Pesq. Vet. Bras. 30(4):301-304, abril 2010

\title{
Valores de referência e influência da idade no eritrograma de bubalinos da raça Murrah ${ }^{1}$
}

\author{
Viviani Gomes ${ }^{2,3 *}$, Jéssica A. Moura ${ }^{3}$, Karina M. Madureira ${ }^{3}$, Fernanda \\ Baptistella $^{3}$, Sandra S. Kitamura ${ }^{2}$, Maiara G. Blagitz ${ }^{2}$ e Fernando J. Benesi ${ }^{2}$
}

\begin{abstract}
Gomes V., Moura J.A., Madureira K.M., Baptistella F., Kitamura S.S. \& Benesi F.J. 2010. [Reference values and influence of age on erythrogram of Murrah buffaloes.] Valores de referência e influência da idade no eritrograma de bubalinos da raça Murrah. Pesquisa Veterinária Brasileira 30(4):301-304. Departamento de Clínica Médica, Faculdade de Medicina Veterinária e Zootecnia, Universidade de São Paulo, Av. Prof. Dr. Orlando Marques de Paiva 87, São Paulo, SP 05508-270, Brazil. E-mail: vivvet@yahoo.com.br

Erythrograms aid the veterinary practitioner in the diagnosis of diseases that affect domestic animals. However, hematological studies involving buffaloes are scarce, with little information in the literature on reference values for blood components in this species. Therefore, the objective of this study was analyze erythrograms of Murrah breed buffaloes of different ages, both males and females. Buffaloes in the study were distributed into four experimental groups, according to their ages: Group 1, animals from birth to 3 months of age $(n=15)$; Group 2, animals from 4 to 6 months of age $(n=50)$; Group 3, animals from 7 to 12 months of age ( $n=50)$; and Group 4, animals from 13 months to five years of age $(n=50)$. The following values were obtained, for Group 1: $7.9 \times 10^{6}$ erythrocytes $/ \mathrm{mL}(\mathrm{He}), 13.0 \mathrm{~g} / \mathrm{dL}$ hemoglobin $(\mathrm{Hb})$, hematocrit $(\mathrm{Ht}) 38.9 \%$, Mean Corpuscular Volume (MCV) $49.0 \mathrm{fl}$, Mean Hemoglobin Corpuscular Concentration (MCHC) 33.6\%, and Mean Corpuscular Hemoglobin (MCH) 16.4 pg; for Group 2: He7.1x10\%/mL, Hb12.5g/dL, Ht 36.8\%, MCV 52.4 fl; MCHC 33.9\%; and $\mathrm{MCH} 17.8 \mathrm{pg}$; for Group 3: He 7.9×10\%/mL, Hb12.0 $/ \mathrm{dL}, \mathrm{Ht} 33.8 \%$, MCV $43.1 \mathrm{fl}$, MCHC 35.4\%, and MCH 15.34pg; for Group 4: He 6.7×106/mL, Hb 11.7g/dL, Ht 34.4\%, MCV $53.4 \mathrm{fl}, \mathrm{MCHC} 34.4 \%$, and $\mathrm{MCH} 17.4 \mathrm{pg}$. Statistical analysis of the results for the different age ranges permitted the conclusion that when the animals got older there was a decrease in the mean red cell counts $(\mathrm{He})$, hemoglobin concentration $(\mathrm{Hb})$ and hematocrit values $(\mathrm{Ht})$. Although hematometric indexes showed significant variations in G3.
\end{abstract}

INDEX-TERMS: Age, erythrocytes, hemoglobin, hematocrit, Murrah buffaloes.

RESUMO.- O eritrograma é uma avaliação que representa um auxílio ao clínico no diagnóstico de afecções que acometem os animais domésticos. No entanto, estudos hematológicos em bubalinos são escassos, havendo poucas informações na literatura a respeito de valores de referência dos constituintes sanguíneos desta espécie. As-

\footnotetext{
${ }^{1}$ Recebido em 1 de outubro de 2008.

Aceito para publicação em 8 de setembro de 2009.

${ }^{2}$ Departamento de Clínica Médica, Faculdade de Medicina Veterinária e Zootecnia, Universidade de São Paulo, Av. José Antunes de Lisboa 840, Leme, SP 13613-130, Brasil. "Autor para correspondência: viv_vet@yahoo.com.br

${ }^{3}$ Faculdade de Medicina Veterinária da Anhanguera Educacional, Rua Waldemar Silenci 340, Cidade Jardim, Leme, SP 13614-370.
}

sim, este trabalho teve como objetivo a análise do eritrograma de búfalos de diferentes faixas etárias da raça Murrah, machos e fêmeas. Os animais em estudo foram distribuídos em quatro grupos experimentais, de acordo com as idades: Grupo 1: animais com idade entre o nascimento e 3 meses ( $n=15)$; Grupo 2: animais com idade entre 4 e 6 meses ( $n=50)$; Grupo 3: animais com idade entre 7 e 12 meses ( $n=50)$; e Grupo 4: animais com idade superior a 12 meses $(n=50)$. Como resultados das análises foram encontrados os seguintes valores médios, no

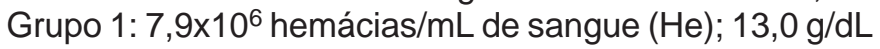
de hemoglobina $(\mathrm{Hb})$; hematócrito $(\mathrm{Ht})$ de $38,9 \%$; Volume Corpuscular Médio (VCM) de 49,0fl; Concentração Hemoglobínica Corpuscular Média (CHCM) de 33,6 \%, e 
Hemoglobina Corpuscular Média (HCM) de 16,4 pg; no Grupo 2: He: 7,1 x106/mL; Hb: $12,5 \mathrm{~g} / \mathrm{dL}$; Ht: 36,8\%; VCM: 52,4 fl; CHCM: 33,9\%, e HCM: 17,8 pg; no Grupo 3: He: 7,9 ×106/mL; Hb: $12,0 \mathrm{~g} / \mathrm{dL}$; Ht: 33,8\%; VCM: 43,1 fl; CHCM: 35,4\%, e HCM: 15,34 pg; e no Grupo 4: He: 6,7 x106/mL; Hb: 11,7 g/dL; Ht: 34,4\%; VCM: 53,4 fl; CHCM: $34,4 \%$. e HCM: $17,4 \mathrm{pg}$. A análise estatística dos resultados encontrados para as diferentes faixas etárias permitiu concluir que ocorreram variações determinadas pela evolução da idade, caracterizadas por diminuição do número médio de hemácias $(\mathrm{He})$, da concentração de hemoglobina $(\mathrm{Hb})$ e do valor do hematócrito $(\mathrm{Ht})$. Os índices hematimétricos apresentaram variações significativas no G3.

TERMOS DE INDEXAÇÃO: Búfalos, eritrograma, faixa etária.

\section{INTRODUÇÃO}

Apesar do histórico da entrada de bubalinos no Brasil ter sido datado como do final do século XIX, as informações específicas sobre a sanidade destes animais ainda são escassas (Oliveira 1997, Ciaramella et al. 2005, Bernarde 2007). Acredita-se que estão sujeitos às mesmas enfermidades que acometem os bovinos (Barbosa et al. 2005), porém para a elucidação da evolução, do diagnóstico e para a diferenciação das doenças há necessidade do conhecimento das alterações clínicas e laboratoriais. Entretanto, para que esses objetivos possam ser alcançados e utilizados na sua plenitude, é fundamental o conhecimento dos valores de referência do hemograma dos animais sadios, bem como os fatores causadores de suas variações (Birgel Júnior et al. 2001).

$\mathrm{Na}$ análise da bibliografia especializada poucas foram as pesquisas dedicadas exclusivamente à determinação do quadro hematimétrico de bubalinos (Vogel \& Vogel 1967, Birgel et al. 1969, Alencar Filho et al. 1972, Jain 1982, Machado et al. 1982, Silva 1986, Ciamarella et al. 2005, Akhtar et al. 2007), observando-se necessidade de maiores estudos sobre o tema, destacando-se que na maioria dos trabalhos encontrados não se avaliou a influência de fatores fisiológicos sobre os constituintes sangüíneos, tais como o padrão racial, a faixa etária e a sanidade dos animais estudados. Assim sendo, esta pesquisa visou o estabelecimento de valores de referência e a avaliação da influência dos fatores etários sobre as variações dos elementos do eritrograma de bubalinos da raça Murrah criados no Estado de São Paulo.

\section{MATERIAL E MÉTODOS}

Foram selecionados 165 bubalinos, sendo 39 animais machos e 126 fêmeas, da raça Murrah, clinicamente sadios, criados extensivamente em 4 (quatro) propriedades leiteiras do Município de Analândia, Estado de São Paulo. Os critérios adotados para o exame físico foram aqueles estabelecidos por Dirksen et al. (1993).

Os animais foram selecionados e distribuídos, segundo a idade, em quatro grupos experimentais: Grupo 1, animais com idade entre o nascimento e 3 meses $(n=15)$; Grupo 2, animais entre 4 e 6 meses ( $n=50)$; Grupo 3, entre 7 e 12 meses $(n=50)$; e Grupo 4, 13 meses a 05 anos de idade $(n=50)$.

A influência da covariável sexo sobre os componentes hematológicos de bubalinos não foi realizada, pois pesquisas em bovinos mostraram que esta covariável não influencia estes parâmetros (Ayres et al. 2001, Silva et al. 2005). Além disso, o número de machos era escasso, nas criações avaliadas, impedindo a formação de grupos homogêneos. Os grupos G1, G2, G3 e G4, respectivamente, foram compostos por 2 machos e 13 fêmeas; 18 machos e 32 fêmeas; 19 machos e 31 fêmeas; e G4 com 50 fêmeas.

O estágio gestacional das fêmeas não foi considerado nesta pesquisa, pois as propriedades estudadas não possuíam tronco de contenção, impossibilitando a realização de palpação retal e identificação das fêmeas gestantes.

As amostras de sangue foram colhidas, ao longo dos anos de 2006 e 2007, através da punção da veia jugular externa, sem garroteamento excessivo do vaso, utilizando o sistema vacutainer $^{\circledR}$, em tubos siliconizados, com capacidade de $5 \mathrm{~mL}$ de sangue, contendo anticoagulante (etilenodiamino-tetracéticodi-potássica, EDTA). As amostras foram mantidas em recipiente de isopor com gelo, desde o transporte até o laboratório, e em seguida acondicionadas em geladeira até o momento da realização dos exames, sempre antes de decorridas 24 horas da colheita.

As amostras de sangue foram submetidas a exames laboratoriais para determinação dos diferentes constituintes do eritrograma, segundo as técnicas descritas por Birgel et al. (1982). A contagem do número de hemácias $(\mathrm{He})$ foi realizada manualmente em hemocitômetro (câmara de Neubauer modificada), sendo os resultados apresentados em milhões $\left(10^{6}\right)$ de células $/ \mathrm{mL}$. A determinação do hematócrito $(\mathrm{Ht})$ foi feita por microtécnica, utilizando-se tubos capilares de vidro, com resultados expressos em porcentagem (\%). Para a dosagem do teor de hemoglobina $(\mathrm{Hb})$, utilizou-se o método da cianometahemoglobina, com os resultados expressos em grama por decilitro ( $\mathrm{g} / \mathrm{dL})$.

Os índices hematimétricos absolutos foram calculados a partir dos valores obtidos para a contagem de hemácias $(\mathrm{He})$, determinação do hematócrito $(\mathrm{Ht})$ e dosagem de hemoglobina $(\mathrm{Hb})$, sendo obtidos o Volume Corpuscular Médio (VCM), em fentolitros (fL); Hemoglobina Corpuscular Média (HCM), expressa em picogramas (pg); e a Concentração de Hemoglobina Corpuscular Média (CHCM), em porcentagem (\%).

Os constituintes do eritrograma apresentaram distribuição paramétrica, segundo o teste de normalidade de KolmogorovSmirnov. Foram calculadas as médias aritméticas, os desviospadrão, e a comparação entre médias dos grupos etários foi determinada pelo teste de Tukey (Sampaio, 1998), usando o software estatístico Minitab (2000).

\section{RESULTADOS}

Os valores das determinações dos constituintes do eritrograma de bubalinos da raça Murrah, considerando a estratificação etária estão apresentados nos Quadros 1 e 2.

$\mathrm{O}$ número médio de hemácias $(\mathrm{He})$ no sangue de bubalinos Murrah diminuiu gradativamente e de forma significativa quando se comparou animais do Grupo $1 \mathrm{com}$ aqueles do Grupo 2 e do Grupo 4.

Os teores médios de hemoglobina $(\mathrm{Hb})$ evidenciaram pequenas variações com a faixa etária, caracterizando-se 
Quadro 1. Bubalinos da raça Murrah, distribuídos por faixa etária, segundo o número absoluto de animais (n) e valores médios e desvios-padrão do número de hemácias (He), teor de hemoglobina $(\mathrm{Hb})$, hematórito $(\mathrm{Ht})$ no sangue

\begin{tabular}{ccccc}
\hline Grupos & $\mathrm{n}$ & $\mathrm{He}\left(\times 10^{6} / \mu \mathrm{L}\right)$ & $\mathrm{Hb}(\mathrm{g} / \mathrm{dL})$ & $\mathrm{Ht}(\%)$ \\
\hline 1 (até 3 meses) & 15 & $8,2 \pm 1,0^{\mathrm{a}}$ & $13,5 \pm 0,9^{\mathrm{a}}$ & $40,6 \pm 3,2^{\mathrm{a}}$ \\
2 (4-6 meses) & 50 & $7,1 \pm 0,9^{\mathrm{b}}$ & $12,5 \pm 1,2^{\mathrm{a}}$ & $36,8 \pm 3,5^{\mathrm{b}}$ \\
3 (7-12 meses) & 50 & $7,9 \pm 1,2^{\mathrm{a}}$ & $12,0 \pm 1,2^{\mathrm{ab}}$ & $33,8 \pm 3,2^{\mathrm{c}}$ \\
4 (1 a 5 anos) & 50 & $6,7 \pm 1,7^{\mathrm{b}}$ & $11,7 \pm 1,8^{\mathrm{b}}$ & $34,4 \pm 3,4^{\mathrm{c}}$ \\
Significância & & $\mathrm{P}<0,0001$ & $\mathrm{P}<0,001$ & $\mathrm{P}<0,0001$
\end{tabular}

Letras diferentes na mesma coluna indicam diferença estatística significativa.

Quadro 2. Bubalinos da raça Murrah, distribuídos por faixa etária, segundo o número absoluto de animais (n) e valores médios e desvios-padrão dos índices hematimétricos absolutos: Volume Corpuscular Médio (VCM), Concentração Hemoglobínica Corpuscular Média (CHCM), e Hemoglobina Corpuscular Média (HCM) no sangue

\begin{tabular}{ccccc}
\hline Grupos & $\mathrm{n}$ & VCM (fl) & CHCM (\%) & HCM $(\mathrm{pg})$ \\
\hline 1 (até 3 meses) & 15 & $50,1 \pm 5,7^{\mathrm{a}}$ & $33,8 \pm 3,7^{\mathrm{ab}}$ & $16,4 \pm 1,6^{\mathrm{ab}}$ \\
2 (4-6 meses) & 50 & $52,4 \pm 6,4^{\mathrm{a}}$ & $33,9 \pm 2,3^{\mathrm{b}}$ & $17,8 \pm 2,0^{\mathrm{a}}$ \\
3 (7-12 meses) & 50 & $43,1 \pm 4,3^{\mathrm{b}}$ & $35,4 \pm 2,5^{\mathrm{a}}$ & $15,34 \pm 1,7^{\mathrm{b}}$ \\
4 (1 a 5 anos) & 50 & $53,4 \pm 9,1^{\mathrm{a}}$ & $34,4 \pm 2,3^{\mathrm{ab}}$ & $17,4 \pm 4,6^{\mathrm{a}}$ \\
Significância & & $\mathrm{P}<0,0001$ & $\mathrm{P}<0,029$ & $\mathrm{P}<0,0001$
\end{tabular}

Letras diferentes na mesma coluna indicam diferença estatística significativa.

certa estabilidade até um ano de vida, quando diminuíram nos animais com idade superior a 12 meses $(11,7 \mathrm{~g} / \mathrm{dL})$.

Os valores do hematócrito $(\mathrm{Ht})$ sofreram influência de fatores etários, sendo significativamente maiores nos animais jovens, com um valor máximo $(40,6 \%)$ no Grupo 1 , para a seguir diminuírem gradativamente até atingirem valores mínimos nos animais do Grupo 3 (33,8 \%), apresentando estabilidade a partir do sétimo mês de idade.

Para os valores médios do VCM observou-se certa estabilidade nos animais com idade de 1-6 meses, seguida por queda significativa entre 7 e 12 meses e retorno aos valores iniciais a partir dos 12 meses de idade.

Os valores médios do CHCM, apesar de apresentarem variação significativa, não evidenciaram um comportamento bem definido, nas diferentes faixas etárias estudadas, comportamento similar foi observado para o HCM.

\section{DISCUSSÃO}

Dentre os estudos sobre hematologia de bubalinos (Vogel \& Vogel 1967, Birgel et al. 1969, Alencar Filho et al. 1972, Jain 1982, Machado et al. 1982, Silva 1986, Ciamarella et al. 2005, Akhtar et al. 2007), apenas Jain (1982) e Silva (1986) estudaram a espécime da raça Murrah, e somente Birgel et al. (1969) e Silva (1986) avaliaram a influência da idade sobre os constituintes do eritrograma, verificando diminuição nos valores do número de hemácias, da taxa de hemoglobina e do hematócrito com a evolução da idade, comportamento concordante com o encontrado na presente pesquisa.
Os valores médios de $\mathrm{He}, \mathrm{Hb}$ e $\mathrm{Ht}$ encontrados por Birgel et al. (1969) foram maiores que os observados neste trabalho, nos animais com faixa etária inferior a 12 meses, podendo tal fato ser atribuído às condições de manejo a que estes animais foram submetidos, assim como às técnicas utilizadas para as determinações de $\mathrm{Hb}$ e Ht, ou seja, os métodos da hematina ácida com o uso hemoglobinômetro de Sahli e macro-hematócrito de Wintrobe.

Machado et al. (1982) estudaram o número de hemácias e taxa de hemoglobina em 12 bezerros búfalos da raça Mediterrâneo e observaram valores médios de $8,29 \times 10^{6} / \mathrm{mL}$ e $13,24 \mathrm{~g} / \mathrm{dL}$, respectivamente, sendo estes semelhantes aos encontrados nesta pesquisa para os animais do Grupo 1, apesar da diferença racial. Da mesma forma, o valor médio do hematócrito determinado pelo macro-método foi similar ao encontrado para o Grupo 2.

Os teores médios de hemoglobina encontrados por Silva (1986) foram de 13,22; 12,64; 14,52; 11,73; 11,40; $12,26 \mathrm{~g} / \mathrm{dL}$ em animais com idade de 1-4 meses, 5-8 meses, 9-13 meses, 13-24 meses e 25-72 meses, respectivamente, observando oscilações não encontradas na presente pesquisa.

Silva (1986) encontrou valores médios de He menores aos encontrados nesta investigação para animais adultos, enquanto Alencar Filho et al. (1972) observaram maiores valores, provavelmente em função do manejo entre as propriedades estudadas.

Os valores médios de $\mathrm{Hb}$ encontrados no sangue de bubalinos do Grupo 4 foram semelhantes aos encontrados por Jain (1982), Silva (1986) e Aktar (2007). Porém menores que os observados por Vogel \& Vogel (1967), Birgel et al. (1969) e Ciamarella et al. (2005), provavelmente devido à diferenciação racial e metodologias utilizadas. Vogel \& Vogel (1967) e Birgel et al. (1969) não especificaram as raças utilizadas, e apenas Birgel et al. (1969) considerou as faixas etárias estudadas, e Ciamarella et al. (2005) utilizaram animais da raça Mediterrâneo. Birgel et al. (1969) utilizou o método da hematina ácida com hemoglobinômetro de Sahli, diferente do método da cianometahemoglobina utilizada nesta pesquisa. Os valores médios de $\mathrm{Hb}$ encontrados por Alencar Filho (1972) foram maiores que os encontrados, porém o autor estudou animais da raça Jafarabadi.

$\mathrm{O}$ valor médio de Ht determinado nesta pesquisa para animais do Grupo 4 foram semelhantes aos encontrados por Alencar Filho (1972), Jain (1982), Ciamarella et al. (2005) e Aktar et al. (2007), e menores que os demonstrados por Vogel \& Vogel (1967) e Birgel et al. (1969), provavelmente pelas diferenças raciais e metodológicas entre as pesquisas. Vogel \& Vogel (1968) determinaram o hematócrito pelo macro-método de Wintrobe.

Foram encontradas poucas citações a respeito dos valores dos índices hematimétricos absolutos, pois os mesmos dependem da realização do eritrograma completo, fato não realizado pela maioria dos autores, além do que, os poucos resultados encontrados apresentaram grandes os- 
cilações, dificultando as comparações entre os mesmos e a presente investigação; apesar de não ter se encontrado oscilações entre os Grupos 1, 2 e 4, apenas quando os mesmos foram comparados com o Grupo 3.

Ciaramella et al. (2005) analisaram o perfil hematológico de 100 bubalinos sadios da raça Mediterrâneo e observaram valores médios de VCM semelhantes aos encontrados nesta pesquisa, porém o HCM foi inferior, fato que pode ser atribuído à raça dos animais estudados, ou decorrentes das diferenças entre as técnicas usadas para a dosagem de $\mathrm{Hb}$, que influencia na determinação do HCM, pois Ciamarella et al. (2005) utilizaram equipamento automático para as mensurações de $\mathrm{Hb}$, e não descreveram a metodologia utilizada.

\section{CONCLUSÕES}

Com base nos resultados obtidos foi possível determinar os valores de referência para os constituintes do eritrograma de animais da espécie bubalina, e verificar variações determinadas pela evolução da idade entre as faixas etárias estudadas, caracterizadas por diminuição do número médio de hemácias $(\mathrm{He})$, da concentração de hemoglobina $(\mathrm{Hb})$ e no valor do hematócrito $(\mathrm{Ht})$.

Os índices hematimétricos VCM e HCM foram menores nos animais pertencentes ao $\mathrm{G} 3$ e o $\mathrm{CHCM}$ foi maior no retro-referido grupo.

As oscilações encontradas de acordo com as faixas etárias estudadas são importantes parâmetros a serem utilizados na interpretação do eritrograma de animais da espécie bubalina e no diagnóstico das enfermidades que acometem estes animais.

\section{REFERÊNCIAS}

Alencar Filho R.A., Saad A.D., Oliveira A.R. \& Lida L. 1972. Quadro hematimétrico de búfalos. Biológico, São Paulo, 38:131-135.

Akhtar M.Z., Khan A., Khan M.Z. \& Muhammad G. 2007. Haematobiochemical aspects of parturient haemoglobinuria in bufallo. Turk $\mathrm{J}$. Vet. Anim. Sci. 31(2):119-123.

Ayres M.C.C., Birgel E.H., D’Angelino J.L. \& Benesi F.J. 2001. Avaliação da influência de fatores relacionados ao sexo sobre a variabilidade do eritrograma de zebuínos (Bos indicus, Linnaeus, 1758) da raça Nelore, criados no estado de São Paulo. Revta Bras. Saúde Prod. Anim. 1(2):31-36.
Barbosa J.D., Oliveira C.M.C., Duarte M.D. \& Siveira J.A.S. 2005. Doenças de búfalos na Amazônia. Proc. $2^{\circ}$ Simpósio Mineiro de Buiatria, Belo Horizonte.

Bernarde O. 2007. Bubalinocultura no Brasil: situação e importância econômica. Revta Bras. Reprod. Anim. 31(3):293-298.

Birgel E.H., Araujo L.M., Amaral V. \& Gomes M.C.G. 1969. Quadro hematimétrico de búfalos criados na região noroeste do Estado de São Paulo. Ciência e Cultura 21:427-428.

Birgel E.H., Larsson M.H.M.A., Hagiwara M.K., Vasconcellos S.A., Larsson C.E., Ogassawara S. \& Benesi F.J. 1982. Patologia Clínica Veterinária. Sociedade Paulista de Medicina Veterinária, São Paulo, p.177-213.

Birgel Júnior E.H., D’Angelino J.L., Benesi F.J. \& Birgel E.H. 2001. Valores de referência do eritrograma de bovinos da raça Jersey criados no Estado de São Paulo. Arq. Bras. Med. Vet. Zootec. 53(2):1-9.

Ciaramella P., Corona M., Ambrosio R., Consalvo F. \& Persechino A. 2005. Haematological profile on non lactating Mediterranean buffaloes (Bubalus bubalis) ranging in age from 24 months to 14 years. Res. Vet. Sci. 70:77-80.

Dirksen G., Gründer H.-D. \& Stöber M. 1993. Exame Clínico dos Bovinos. $3^{\mathrm{a}}$ ed. Guanabara Koogan, Rio de Janeiro. 419p.

Jain N.C. 1982. Haematological studies on normal lactating Indian water buffaloes. Res. Vet. Sci. 32:52-56.

Machado M.A., Kohayagama A., Guimarães J., Oba E. \& Vilares J.B. 1982. Estudo sobre vários constituintes hematológicos em bubalinos confinados até um ano de idade. Proc. 18ํㅡㄹ. Congr. Bras. Med. Vet., Camboriú, SC. 272p.

Minitab 2000. The Student Edition of Minitab Statistical software adapted for education, 13.0 Release. User's Manual. Addison-Wesley, New York.

Oliveira J.F.S. 1997. Sistema de criação de bubalinos, p.199-217. In: Oliveira G.J.C., Almeida, A.M.L. \& Filho U.A.S. (Eds), O Búfalo no Brasil. Universidade Federal da Bahia, Cruz das Almas.

Sampaio I.B.M. 1998. Estatística Aplicada à Experimentação Animal. Universidade Federal de Minas Gerais, Belo Horizonte. 221p.

Silva M.B. 1986. Contribuição ao quadro eritrocitário de bubalinos fêmeas das raças Jafarabadi, Murrah e Mediterrânea criados no estado de São Paulo. Dissertação de Mestrado em Clínica Médica, Universidade de São Paulo, São Paulo. 115p.

Silva R.M.N., Souza B.B., Souza A.P., Marinho M.L., Tavares G.P. \& Silva E.M.N. 2005. Efeito do sexo e da idade sobre os parâmetros fisiológicos e hematológicos de bovinos da raça Sindi no semi-árido. Ciência Agrotécnica 29(1):193-199.

Vogel L. \& Vogel J. 1967. Alguns índices hematimétricos de Bubalus bubalis. Veterinária, Rio de J., 20:181-186.

Vogel L. \& Vogel J. 1968. Determinação dos índices eritrocitários de Wintrobe em Bubalus bubalis. Revta Med. Vet. 4:21-24. 